\title{
Avaliação da Acessibilidade de Regiões Urbanas de Curitiba aos Serviços de Saúde Pública Baseada em Análise de Grafos e Georreferenciamento
}

\author{
Clauciane Lima ${ }^{1}$, Altieris M. Peixoto ${ }^{1}$, \\ Luiz Gomes-Jr ${ }^{1}$, Keiko V. O. Fonseca ${ }^{1}$, Ricardo Lüders ${ }^{1}$ \\ ${ }^{1}$ Universidade Tecnológica Federal do Paraná (UTFPR) - Curitiba - Brazil \\ claucylima@hotmail.com, altieris.marcelino@gmail.com \\ \{lcjunior, keiko, luders\}eutfpr.edu.br
}

\begin{abstract}
Resumo. Este artigo descreve uma abordagem baseada em análise de grafos e georreferenciamento para determinar medidas de acessibilidade de regiões urbanas às unidades de saúde, utilizando dados abertos de serviços de saúde e transporte públicos de Curitiba. Um bom atendimento primário de saúde pode evitar complicações e reduzir consequências das doenças, além de considerar questões sociais de atendimento da população. A acessibilidade foi medida para deslocamentos a pé e por transporte público. Estas medidas são agregadas em um modelo que considera fatores socioeconômicos para listar zonas de maior vulnerabilidade, as quais devem ser priorizadas em termos de investimento público ou ajustes na rede de transporte. Os resultados mostram que o modelo utilizado é efetivo na identificação de regiões vulneráveis.
\end{abstract}

\section{Introdução}

O acesso eficiente às unidades de saúde propiciado pelo transporte público pode favorecer a busca por atendimento preventivo a doenças. Porém, muitas cidades brasileiras não possuem serviço de transporte público de qualidade [Krempi 2004]. Assim, administradores públicos necessitam de informações confiáveis para identificar regiões com problemas de acesso e direcionar recursos à população mais vulnerável. A acessibilidade espacial à saúde refere-se à facilidade com que a população de uma determinada área consegue chegar aos postos de atendimento médico [Hewko et al. 2002]. A acessibilidade espacial enfatiza o papel da distância geográfica nas interações entre serviços de saúde e demandas populacionais [Luo and Wang 2003]. Medidas de acessibilidade espacial à saúde têm recebido atenção devido à sua capacidade de levar em conta variações geográficas dentro de grandes regiões. Uma medida simples de acessibilidade espacial pode ser a distância ou o tempo de viagem de uma pessoa até o serviço de saúde mais próximo [Maon and Nekorchu 2013]. Um método que avalia a eficácia e eficiência dos equipamentos públicos foi proposta em [Raia and Pereira 2001]. Como resultado, obtevese uma avaliação da localização espacial dos serviços de saúde para Bauru/SP.

Este artigo tem como foco a avaliação de medidas de acessibilidade física a unidades de saúde de Curitiba, levando em conta o nível de renda dos habitantes e identificando regiões vulneráveis da cidade. Foram utilizados dados abertos contendo informações sobre a malha de transporte público e unidades de saúde de Curitiba. Os dados foram usados para a construção de um grafo a partir do qual se estimou o tempo de deslocamento 
dos usuários do transporte público nas regiões identificadas (Seção 2). As estimativas de tempo foram então agregadas a dados socioeconômicos para a construção de um modelo de detecção de outliers para identificação de regiões prioritárias para investimentos (Seção 3). Ou seja, regiões com populações que dependem do transporte público, mas estão sujeitas a tempos elevados de deslocamento.

\section{Preparação e processamento dos dados}

Este trabalho considera a estimativa do tempo despendido no deslocamento de um usuário do serviço de saúde desde um ponto de ônibus de origem (em geral próximo de sua residência) até a unidade de saúde mais próxima na qual o usuário está cadastrado. Este cadastro é função do seu endereço residencial dentro de uma região predefinida da cidade denominada regional. Os pontos de ônibus são utilizados como local de origem dos usuários, pois os dados das residências não são abertos. Isso é uma aproximação do que ocorre de fato, uma vez que o trajeto da residência até o ponto de ônibus mais próximo não é considerado. O custo de deslocamento é então representado pelo tempo de deslocamento do ponto de ônibus de origem até o ponto de ônibus mais próximo da unidade de saúde correspondente, somado ao tempo de caminhada a pé deste ponto até a unidade.

Para cada ponto de origem, apenas unidades de saúde na mesma regional são consideradas na identificação da rota mais curta. Isso ocorre para evitar distorções em áreas de fronteira entre regionais, onde eventualmente seria melhor atender o residente de uma regional em outra. A rota mais curta é calculada considerando-se a malha da rede de transporte obtida a partir do portal URBS (Urbanização de Curitiba) http://dadosabertos.c3sl.ufpr.br/curitibaurbs/. Esta malha é transformada em um grafo no qual vértices representam pontos de ônibus e arestas representam conexões entre pontos devidas às linhas de ônibus. O peso das arestas é determinado pela distância entre os pontos. O grafo foi construído no banco de grafos Neo4J (https://neo4j.com/) e as rotas foram calculadas pelo algoritmo de Dijkstra usando uma consulta Cypher. Para transformar as distâncias de deslocamento em estimativas de tempo, foram consideradas as distâncias $d_{o}$ (obtida na rede de transporte pelo caminho mínimo entre dois pontos de ônibus) e $d_{c}$ (obtida para um pedestre caminhando em linha reta entre ponto e destino final). Essas distâncias são percorridas com velocidades $v_{o}=19 \mathrm{~km} / \mathrm{h}$ (ônibus) e $v_{c}=4,8 \mathrm{~km} / \mathrm{h}$ (caminhada), respectivamente, segundo [Miranda and Silva 2012] e [Garcia and Raia 2015]. O tempo de viagem $t_{v}=d_{c} / v_{c}$ para $d_{c} \leq 500 \mathrm{~m} \mathrm{e} t_{v}=d_{o} / v_{o}$ para $d_{o}>500 \mathrm{~m}$ entre a origem e a unidade de saúde mais próxima. Desta forma, usuários localizados em pontos com uma distância menor que $500 \mathrm{~m}$ da unidade de saúde realizam o percurso a pé e de ônibus, caso contrário. O tempo médio de viagem por bairro é obtido pela média dos tempos de viagem $t_{v}$ a partir de cada ponto de ônibus do bairro. Outro dado utilizado na análise foi o rendimento médio do responsável familiar (RMR) em salários mínimos por bairro da cidade. Estes dados são disponibilizados pelo IPPUC (Instituto de Pesquisa e Planejamento Urbano de Curitiba) usando a metodologia de [Garcia and Raia 2015].

\section{Resultados e discussão}

O principal objetivo deste trabalho é estabelecer critérios para a identificação de regiões com acessibilidade inadequada em relação aos serviços de saúde. Uma maneira de se determinar esta situação é usar o tempo médio de deslocamento como critério para identificar regiões prioritárias. Para tanto, foi criado um mapa temático com o tempos médio 
de viagem dos usuários dos bairros de Curitiba, conforme mostrado na Figura 1. Nota-se na Figura 1 que a maioria dos bairros de Curitiba apresentam um tempo médio de viagem de até 5 minutos (de ônibus, ou a pé se a distância for menor do que $500 \mathrm{~m}$ ). No entanto, este critério não considera a diversidade socioeconômica da população.

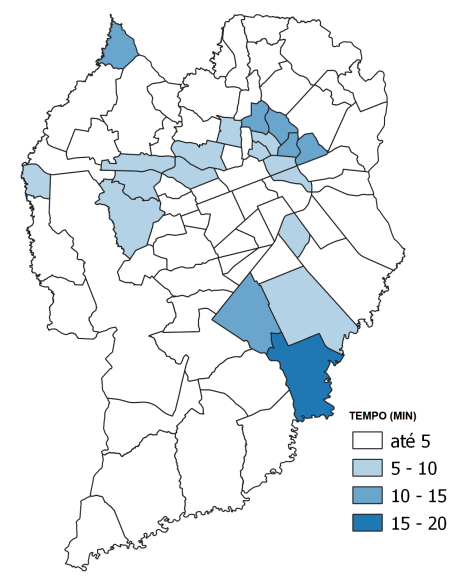

Figura 1. Tempo médio de viagem.

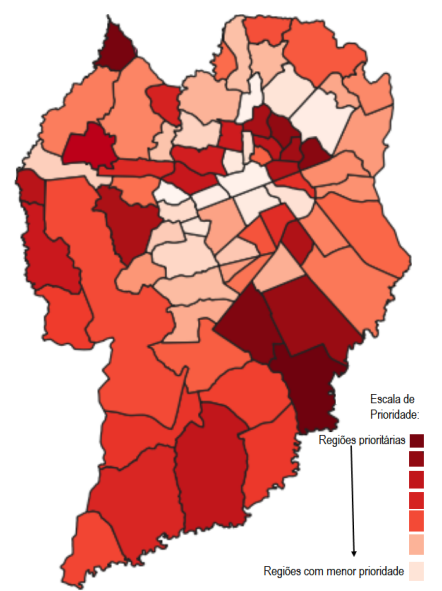

Figura 2. Bairros prioritários.

Para a identificação de regiões prioritárias considerando características da população, adotou-se um modelo de detecção de outliers baseado em regressão linear multivariada [Chandola et al. 2009], conforme a Figura 3.

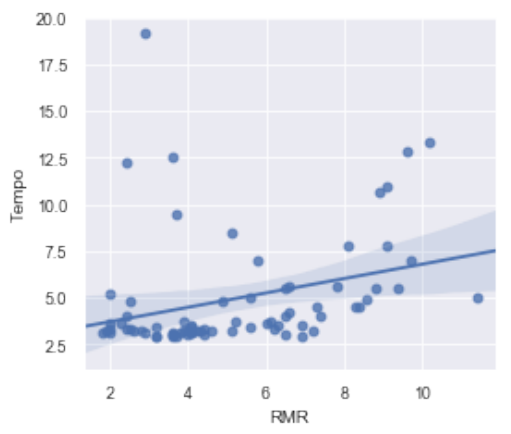

Figura 3. Relação RMR $\times$ tempo.

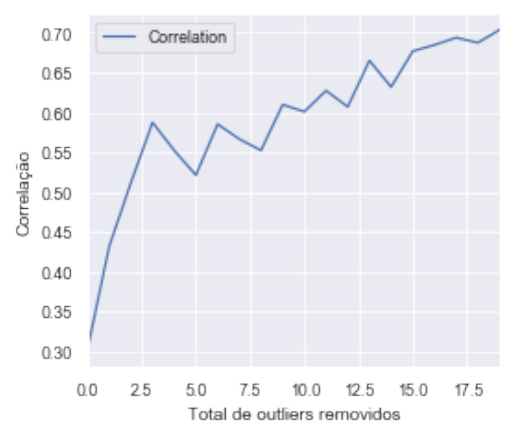

Figura 4. Correlação sem outliers.

Em tarefas de detecção de outliers baseadas em regressão, pontos com valores residuais altos são classificados como outliers. Neste caso, a variável de resposta (eixo y) é o tempo de deslocamento e o preditor (eixo x) é a renda (RMR), ambos referente a média por bairro. Uma particularidade do nosso modelo de detecção de outliers é que só estamos interessados nos outliers com resíduos positivos, ou seja, bairros com tempos elevados para populações que dependem notadamente do transporte público. Os bairros com altos resíduos negativos são bairros em condições privilegiadas de acessibilidade nas suas faixas de renda. A Figura 2 mostra os bairros de Curitiba de acordo com os valores calculados de resíduo. As regiões prioritárias coincidem com regiões de periferia da cidade, mostrando que o modelo produz resultados mais adequados do que a simples análise do tempo de viagem da Figura 1. Intuitivamente, o modelo prevê, para cada nível de renda, o tempo de deslocamento que se esperaria considerando a tendência apresentada nos dados de treinamento. Como o objetivo do modelo é a detecção dos outliers, 
a existência de muitos outliers deteriora a qualidade do modelo, conforme mostrado na Figura 3. A Figura 4 mostra como a adequação do modelo aumenta à medida que outliers são retirados (como se medidas corretivas fossem adotadas pela administração). A correlação entre renda e tempo de viagem inicia em 0,3 considerando todos os dados e aumenta à medida que outliers são retirados, chegando a 0,7 de correlação após a retirada de 20 outliers. Obviamente, a retirada dos outliers é uma simplificação. Na prática, o modelo seria reconstruído com novos tempos de deslocamento da região melhorada.

\section{Conclusão}

Este artigo apresentou uma análise da acessibilidade de diferentes regiões de Curitiba às unidades de saúde. A partir de informações geográficas e um modelo de grafo, foram estabelecidas métricas que capturam o custo de deslocamento da população às unidades de saúde. Informações socioeconômicas foram agregadas para a criação de um modelo de detecção de outliers que identifica regiões prioritárias. O modelo proposto usa resíduos de uma regressão linear para identificar os outliers significativos. Assim, a maior adequação do modelo aos dados, quando os outliers são retirados, demonstra como ações do poder público podem ser direcionadas para regiões prioritárias. Essas regiões apresentam forte demanda por transporte público, mas são atendidas com elevados tempos de transporte.

Agradecimentos: Este trabalho tem apoio do Projeto Smart City Concepts in Curitiba, Brasil/Suécia e foi realizado com apoio da Coordenação de Aperfeiçoamento de Pessoal de Nível Superior-Brasil (CAPES) - PROAP.

\section{Referências}

Chandola, V., Banerjee, A., and Kumar, V. (2009). Anomaly detection: A survey. ACM Comput. Surv., 41(3):15:1-58.

Garcia, P. B. M. and Raia, A. A. (2015). Análise da acessibilidade a hospitais: Estudo de caso para as cidades de São Carlos (SP) e Rio Claro (SP). Revista Brasileira de Gestão Urbana, 7:21-47.

Hewko, J., Smoyer-Tomic, K. E., and Hodgson, M. J. (2002). Measuring neighbourhood spatial accessibility to urban amenities: Does aggregation error matter? Environment and Planning A: Economy and Space, 34(7):1185-1206.

Krempi, A. P. (2004). Explorando recursos da estatística espacial para análise da acessibilidade da cidade de Bauru. Dissertação de Mestrado, Escola de Engenharia de São Carlos, Universidade de São Paulo.

Luo, W. and Wang, F. (2003). Measures of spatial accessibility to health care in a GIS environment: Synthesis and a case study in the Chicago region. Environment and Planning B: Planning and Design, 30:865-884.

Maon, L. and Nekorchu, D. (2013). Measuring spatial accessibility to healthcare for populations with multiple transportation modes. Health \& Place, 24:115-122.

Miranda, H. and Silva, A. (2012). Benchmarking sustainable urban mobility : the case of Curitiba, Brazil. Transport Policy, 21:141-151.

Raia, A. A. and Pereira, C. A. (2001). Metodologia para simulação de mapas de equidade e acessibilidade a equipamentos e acessibilidade a equipamentos públicos urbanos. Relatório de pesquisa São Carlos. 